\title{
Variación de la autonomía en el aprendizaje, en función de la gestión del conocimiento, para disminuir en los alumnos los efectos del aislamiento
}

\section{Variation of the autonomy in the learning, in function of the knowledge management, to diminish in the students the effects of the isolation}

\author{
Luis Danilo Flores Rivera \\ Escuela Superior Politécnica de Chimborazo (ESPOCH). Riobamba, Ecuador \\ luis.floresr@espoch.edu.ec \\ Carlos Fernando Meléndez Tamayo \\ Universidad Técnica de Ambato (UTA). Ambato, Ecuador \\ cmelendez77@uta.edu.ec
}

\begin{abstract}
Resumen
El aprendizaje autónomo en la formación universitaria es un elemento clave que permite gestionar el conocimiento, así como también, reforzar y retroalimentar contenidos, es en ese sentido que, los recursos tecnológicos en línea son considerados como el aliado ideal en el fortalecimiento del aprendizaje autónomo, puesto que, facilita los procesos de aprendizaje y disminuye efectos producidos por el aislamiento. Hoy en día el uso y aplicación de un sin número de herramientas tecnológicas virtuales se adaptan a las necesidades específicas del educando.

La presente investigación se desarrolló en una población de 58 estudiantes y 26 docentes de una universidad privada del Ecuador que contaba con equipamiento tecnológico y herramientas en línea adecuadas para efectuar la misma. Se aplicó una encuesta estructurada que permitió identificar y conocer sobre las herramientas en línea en función de las actividades de aprendizaje autónomo, el rendimiento académico, el empleo de los recursos tecnológicos entre otros, de los cuales se pudo determinar la importancia de los recursos en línea en la gestión académica del aprendizaje autónomo del estudiante.
\end{abstract}

Palabras clave

Aprendizaje autónomo; herramientas en línea; recursos tecnológicos; tecnologías de la información y comunicación (TIC), Educación Superior.

\begin{abstract}
Autonomous learning in university education is a key element in managing knowledge, as well as reinforcing and feedback on content. In this sense, online technological resources are considered as the ideal ally in strengthening autonomous learning. Which facilitates learning processes and reduces effects produced by isolation. Today the use and application of a number of virtual technological tools are adapted to the specific needs of the student. This research was carried out in a population of 58 students and 26 teachers from a private university in Ecuador that had the necessary technological equipment and online tools to carry it out. A structured survey was conducted to identify and learn about online tools in terms of autonomous learning activities, academic performance, use of technological resources among others, from which it was possible to determine the importance of online resources in the academic management of autonomous student learning.
\end{abstract}

Key words

Autonomous Learning; online tools; technological resources; Information and Communication Technologies (ICT), Higher Education. 


\section{Introducción}

La educación superior se ha beneficiado del desarrollo tecnológico de las herramientas en línea, que combinadas a estrategias pedagógicas encaminan el perfeccionamiento de aprendizajes de los educandos. Esta apreciación se relaciona con lo señalado por Cataldi, Lage y Cabero (2010) que destacan el uso de la tecnología informática integrada al aprendizaje colaborativo, fomentan la construcción del conocimiento y el desarrollo de competencias profesionales. En efecto a lo indicado, hoy en día son posibles las experiencias de aprendizaje personalizadas que no es más que el aprendizaje adaptativo. (Instituto Tecnológico de Monterrey, 2014)

Es en ese sentido que los estudiantes que ingresan a la universidad ecuatoriana tienen heterogeneidad de conocimientos y evidencian deficiencias en el uso de recursos tecnológicos, mismos que se ven reflejados en el bajo desempeño en las actividades educativas autónomas.

Es por esto que resulta imprecindible el fortalecer y adaptar el uso de las herramientas en línea en el aprendizaje autónomo de los estudiantes universitarios, las mismas que permiten optimizar tiempos y porque no decirlo recursos.

Por otra parte, el estudio del aprendizaje autónomo en la formación universitaria refleja el grado de aceptación y aporte que brindan los recursos tecnológicos a las actividades de aprendizaje autónomo (Flores Rivera, 2015); siendo el estudiante y el docente universitario los actores que perciben esta oportunidad de incrementar y mejorar sus conocimientos con la aplicación de las herramientas en línea.

Los recursos tecnológicos en línea a medida que siguen desarrollándose serán más amigables y versátiles; esto será un plus para que el estudiante aplique la tecnología en línea, en su comprensión y realización de actividades académicas universitarias.

Los objetivos formulados en la investigación son: Conocer el nivel de aplicación de los recursos y herramientas en línea como bibliotecas virtuales, blogs, chat, correo electrónico, foros, plataformas virtuales, redes sociales, repositorios digitales, video conferencias en el aprendizaje autónomo universitario; Identificar los medios por los cuales se difunden los recursos y herramientas en línea que fomenten el aprendizaje autónomo universitario.

Los resultados de la investigación de los actores del proceso aprendizaje autónomo legitiman las competencias que generan los recursos tecnológicos en línea. Adicionalmente los rendimientos académicos comparados, muestran una relación e incremento porcentual de las calificaciones. Esto proyectará una perspectiva para abordar nuevos conocimientos y resolver problemas más complejos que pueden presentarse en la autonomía de la educación. 


\section{Marco Teórico}

\section{Aprendizaje Autónomo}

Al hablar de aprendizaje autónomo es hablar de aprendizaje en general, al cual se le integran características de no depender de personas o que dirijan el mismo (Moreno \& Martínez, 2007). También se indica que el aprendizaje autónomo se le conoce como aprendizaje auto-dirigido, aprendizaje autorregulado y autoaprendizaje. Esta tiene un propósito que es el desarrollo personal, la dirección hacia el interior de uno mismo, la autorrealización y la autonomía (Lobato Fraile, 2006).

El interés y crecimiento en la autonomía como meta educativa se identificó por los sucesos ocurridos en el siglo XX, especialmente por la Segunda Guerra Mundial, cuando las ideas de autonomía y autodirección fueron objeto de análisis y de investigación en las ciencias sociales, psicología, filosofía y ciencias políticas (Finch, 2010). Los cambios en la filosofía educativa, la teoría del aprendizaje de idiomas, las creencias políticas, la necesidad de adaptarse a los cambios rápidos en la tecnología, las comunicaciones y el empleo, estimuló el reconocimiento de que aprender a aprender ahora es más importante que el conocimiento. Se generaron oportunidades por los desarrollos tecnológicos y se amplió la oferta educativa con reducción de costos. (Pemberton, Li, Or, \& Pierson, 1996).

Existen otros factores e ideas que encaminan esta práctica:

1. Movimientos de derechos de las minorías;

2. Una reacción contra el conductismo en la medicina, la política, la música, la poesía, la educación, la psicología, la educación, la filosofía y la lingüística;

3. La aparición de la "autonomía" como un ideal educativo, con una influencia directa en la educación de adultos en Europa;

4. Los avances tecnológicos que contribuyen a la difusión de la autonomía y el auto-acceso;

5. El creciente internacionalismo desde la Segunda Guerra Mundial;

6. Los alumnos adultos y las diferentes necesidades de aprendizaje, dando lugar a programas de aprendizaje flexibles con grados variables de concentración en el aprendizaje y autodirección;

7. La comercialización de servicios lingüísticos, junto con la tendencia de incrementar la responsabilidad de estudiantes como clientes y que estos tomen decisiones conocidas en estas actividades;

8. El aumento de la población escolar y universitaria, que fomente el desarrollo de nuevas estructuras educativas para tratar con un gran número de estudiantes. Siendo útil el aprendizaje auto-dirigido con apoyo institucional en forma de centros de asesoramiento y recursos materiales.

(Gremmo \& Riley, 1995)

El concepto de autonomía individual ha sido central en el pensamiento liberaldemocrático y liberal-humanista europeo desde el siglo XVIII (Lindley, 1986), y fue identificado por Kant como el fundamento de la dignidad humana (Hill, 1991). Holec (1980) observa una tendencia "irreversible" a finales de los años sesenta en los países occidentales industrialmente avanzados que definen un progreso social en términos de

Los recursos tecnológicos en línea fortalecen el aprendizaje autónomo universitario. Luis Danilo Flores Rivera y Carlos Fernando Meléndez Tamayo.

Página 3 de 15 
mejora de la "calidad de vida", dando lugar a diversos tipos de conciencia social, desde la ecología hasta la situación de la mujer, los derechos de los pacientes y la educación.

La educación de adultos (...) se convierte en un instrumento para despertar un creciente sentido de conciencia y liberación en el hombre y, en algunos casos, un instrumento para cambiar el entorno mismo. A partir de la idea del hombre "producto de su sociedad", uno pasa a la idea del hombre "productor de su sociedad". (Holec, 1980).

El aprendizaje autónomo es una competencia que se construye en base a la disciplina y constancia del individuo; estos principios serán la pauta en el logro de nuevos conocimientos. La universidad como entidad que promueve los procesos investigativos, busca en sus estudiantes autonomía para la consecución de sus aprendizajes. La autonomía juega un papel fundamental dentro del contexto de la educación y la formación de las personas, ya que, como lo mencionan Ferroni y Velásquez (2005, s.p.), el aprendizaje es un proceso autónomo donde el estudiante hace apropiación de los conocimientos teóricos y la aplicación de los mismos a partir de la toma de conciencia sobre su responsabilidad en cuándo, cómo y cuánto debe aprender, para saber más y mejor sobre un área o un quehacer específico. La apreciación converge en que los estudiantes universitarios sepan claramente cuándo y por qué aprender (Giné Freixes, 2009, p. 118).

En ese sentido otro concepto relevante, es el que señala (Martínez, 2004, s.p.) al aprendizaje autónomo como un proceso donde el estudiante autorregula su aprendizaje y toma conciencia de sus propios procesos cognitivos y socio-afectivos. Esta toma de conciencia se la llama metacognición. El esfuerzo pedagógico en este caso, se orienta hacia la formación de sujetos centrados en resolver aspectos concretos de su propio aprendizaje, y no sólo en resolver una tarea determinada, es decir, orientar al estudiante a que se cuestione, revise, planifique, controle y evalúe su propia acción de aprendizaje.

Importante lo que menciona Meléndez (2014, p. 86) en el sentido en el que estamos moviéndonos hacia un modelo de enseñanza/aprendizaje en el que el alumno ha de adquirir cada vez mayor autonomía sobre su propio aprendizaje.

Las universidades e instituciones de educación superior (IES) consideran el trabajo autónomo como parte del crédito académico <<unidad de tiempo que valora las actividades académicas que corresponden a los componentes educativos $>$ (CONESUP, 2008), dependiendo la modalidad de estudio (presencial, semipresencial y a distancia) o de los programas académicos, estos factores decidirán en un mayor o menor número de horas de trabajo autónomo (Flores Rivera, 2015). En este ámbito, la autonomía es un requisito y componente esencial que complementa la formación de competencias de una profesión.

\section{Recursos tecnológicos en línea}

Los recursos tecnológicos en línea son parte de las tecnologías de la información y comunicación (TIC), teniendo como antecedente que las TIC, integran y gestionan el hardware (equipos, dispositivos informáticos), software (programas informáticos, aplicaciones) y redes (comunicación de datos empleando el hardware y software).

Los recursos tecnológicos en línea fortalecen el aprendizaje autónomo universitario. Luis Danilo Flores Rivera y Carlos Fernando Meléndez Tamayo.

Página 4 de 15 
En las últimas décadas desde 1985 hasta 2015 se reflejan nuevos enfoques evolutivos y vivencias de las TIC en relación a las máquinas, las posibilidades de interacción que ofrecen medios como la TV y ordenadores que han variado significativamente. A partir de los años noventa la importancia de las comunicaciones, la red y las telecomunicaciones con el surgimiento de Internet. (Grande, Cañón, \& Cantón, 2016).

En la segunda mitad de la década del pasado siglo, la importancia del Hardware y del Software y la aparición del sistema operativo Windows. (Martínez, 1996; GarcíaValcárcel \& Tejedor, 1996; Adell, 1997).

En el siglo XXI, varios autores e instituciones reconocen a las TIC un papel fundamental en la sociedad, expresando su potencial para crear nuevas posibilidades comunicativas, cooperación en el ámbito social, cultural y económico (Cabero, 2001; Majó \& Marqués, 2002; UNESCO, 2002; OCDE, 2002).

La aparición de la Web 2.0 y las redes sociales a principios del siglo XXI, cobran importancia en la comunicación y la gestión de la información lo que se llamó TIC. A esto devino la Sociedad del Conocimiento (UNESCO, 2005).

La inmediatez y dinamismo de la sociedad (Cacheiro González, 2014; Roblizo Colmenero \& Cózar Gutiérrez, 2015), dio paso a la Web 3.0 por medio de los smartphones (Santiago Campión \& Navaridas Nalda, 2012). Hoy en día las TIC se constituyen en herramientas tecnológicas digitales que facilitan la comunicación y la información, dependientes de la accesibilidad e interconexión, promueven mejoras sociales (UNESCO, 2005; Baelo Álvarez \& Cantón Mayo, 2009) supeditadas a intereses económicos (Bautista, 2011; De Pablos, 2007; Majó \& Marqués, 2002).

En ese sentido importante lo señalado por Morrissey (2008), el uso de las TIC contribuye al desarrollo de la creatividad y la inventiva, habilidades que son particularmente valoradas en el mercado laboral. El uso de las TIC como recurso tecnológico es un factor clave para el cambio social. Además señala que la disponibilidad computadores portátiles y dispositivos celulares inteligentes más económicos y eficientes han llevado a una revolución en las comunicaciones entre los jóvenes. Con la interacción social (redes sociales) que se adaptan y personalizan el uso de estas herramientas.

Dentro de las TIC, se tiene un recurso tecnológico en línea esencial y útil en el aprendizaje autónomo como lo es la plataforma virtual (PV), o entorno virtual de aprendizaje (EVA), este escenario integra un conjunto de herramientas, que pueden ser controladas por el tutor o facilitador del aprendizaje y en casos extraordinarios por el administrador del EVA (Flores Rivera, 2015).

En la tabla 1, se indican las principales categorías de las herramientas en línea que emplean docentes y estudiantes en sus actividades académicas.

Los recursos tecnológicos en línea fortalecen el aprendizaje autónomo universitario. Luis Danilo Flores Rivera y Carlos Fernando Meléndez Tamayo. 
Tabla 1

Herramientas de colaboración en línea

\begin{tabular}{|c|c|c|c|}
\hline Categoría & & Tipo de herramienta & Uso en la clase \\
\hline Investigar y buscar & & $\begin{array}{ll}\text { - } & \text { Marcadores } \\
\text { - } & \text { Compartir archivos } \\
\text { - } & \text { Compartir fotografías } \\
\text { - } & \text { Citar fuentes } \\
\end{array}$ & $\begin{array}{l}\text { Docentes y estudiantes guardan, } \\
\text { hacen comentarios, organizan y } \\
\text { comparten recursos de } \\
\text { investigación. }\end{array}$ \\
\hline Escritura colaborativa & & $\begin{array}{ll}\text { - } & \text { Blogs } \\
\text { - } & \text { Documentos colaborativos } \\
\text { (procesador de palabras, hojas } \\
\text { de cálculo, tablas, bases de } \\
\text { datos, gráficos, presentaciones, } \\
\text { entre otros) } \\
\text { - Wikis } \\
\end{array}$ & $\begin{array}{l}\text { Docentes y estudiantes escriben, } \\
\text { comparten y crean de manera } \\
\text { colaborativa contenido en línea. }\end{array}$ \\
\hline $\begin{array}{l}\text { Comunicación } \\
\text { mensajería }\end{array}$ & $\mathrm{y}$ & $\begin{array}{ll}\text { - } & \text { Mensajería instantánea } \\
\text { - } & \text { Microblog } \\
\text { - } & \text { Videoconferencias } \\
\end{array}$ & $\begin{array}{llr}\text { Docentes y estudiantes } & \text { se } \\
\text { comunican entre sí, con otras } \\
\text { clases, miembros de la } \\
\text { comunidad y expertos en el área. }\end{array}$ \\
\hline Creación visual & & $\begin{array}{ll}\text { - } & \text { Dibujo } \\
\text { - } & \text { Creación de imágenes } \\
\text { - } & \text { Edición de imágenes } \\
\text { - } & \text { Mapas mentales/Organizadores } \\
\text { - } & \text { gráficos } \\
\end{array}$ & $\begin{array}{l}\text { Docentes y estudiantes diseñan, } \\
\text { comparten y crean de manera } \\
\text { colaborativa contenido visual en } \\
\text { línea. }\end{array}$ \\
\hline Creación de audio & & $\begin{array}{ll}\text { - } & \text { Creación y edición de audio } \\
\text { - } & \text { Podcasting } \\
\text { - } & \text { Locuciones }\end{array}$ & $\begin{array}{l}\text { Docentes y estudiantes diseñan, } \\
\text { comparten y crean de manera } \\
\text { colaborativa contenido de audio } \\
\text { en línea. }\end{array}$ \\
\hline $\begin{array}{l}\text { Administración } \\
\text { proyecto }\end{array}$ & del & $\begin{array}{ll}\text { - } & \text { Calendarios } \\
\text { - } & \text { Seguimiento de tares e hitos } \\
\text { - } & \text { Listados de tareas pendientes } \\
\end{array}$ & \begin{tabular}{lccc} 
Grupos & de & \multicolumn{2}{c}{ estudiantes } \\
administran & $y$ & organizan & sus \\
proyectos. & & & \\
\end{tabular} \\
\hline Recopilación de datos & & $\begin{array}{ll}\text { - } & \begin{array}{l}\text { Formularios, sondeos } \\
\text { encuestas }\end{array} \\
\text { - } & \text { Mapas socio geográficos } \\
\end{array}$ & $\begin{array}{l}\text { Docentes y estudiantes recopilan } \\
\text { y analizan datos desde una } \\
\text { amplia variedad de recursos. }\end{array}$ \\
\hline Productividad docente & & $\begin{array}{ll}\text { - } & \text { Recursos de evaluación } \\
\text { - } & \text { Calendarios } \\
\text { - } & \text { Recursos para lecciones } \\
\text { - } & \text { Toma de apuntes/asignaciones } \\
\end{array}$ & $\begin{array}{l}\text { Recursos específicos para el } \\
\text { docente ayudan a la organización } \\
\text { y administración de la clase. }\end{array}$ \\
\hline
\end{tabular}

Nota. Tomada de Corporación Intel (2015).

Las herramientas en línea indicadas en la tabla 1, colaboran en la construcción de conocimientos del estudiante; las mismas deben combinarse a estrategias que elabora el docente para el trabajo autónomo.

Importante lo que nos menciona Demetra (2017) en Blackboard blog, en el que indica que la adaptabilidad o el aprendizaje adaptativo tienen como objetivo ofrecer aprendizaje personalizado con mejores resultados al combinar diferentes enfoques, es en ese sentido que, se pueden mencionar varios elementos o factores determinantes en la personalización de aprendizaje: a) el diagnóstico o idea previa es decir, que previamente ha aprendido o conocido, b) medida de interacción en el curso, y finalmente c) el resultado o provecho en función de calificaciones de las actividades propuestas como tareas, evaluaciones o talleres. 
En la figura 1, se ilustran recursos en línea de uso libre y complementario que gestionan un aprendizaje autónomo con característica colaborativa. Estos recursos impulsan la autonomía de aprendizajes y la retroalimentación de conocimientos, así como la adaptabilidad en el proceso educativo.

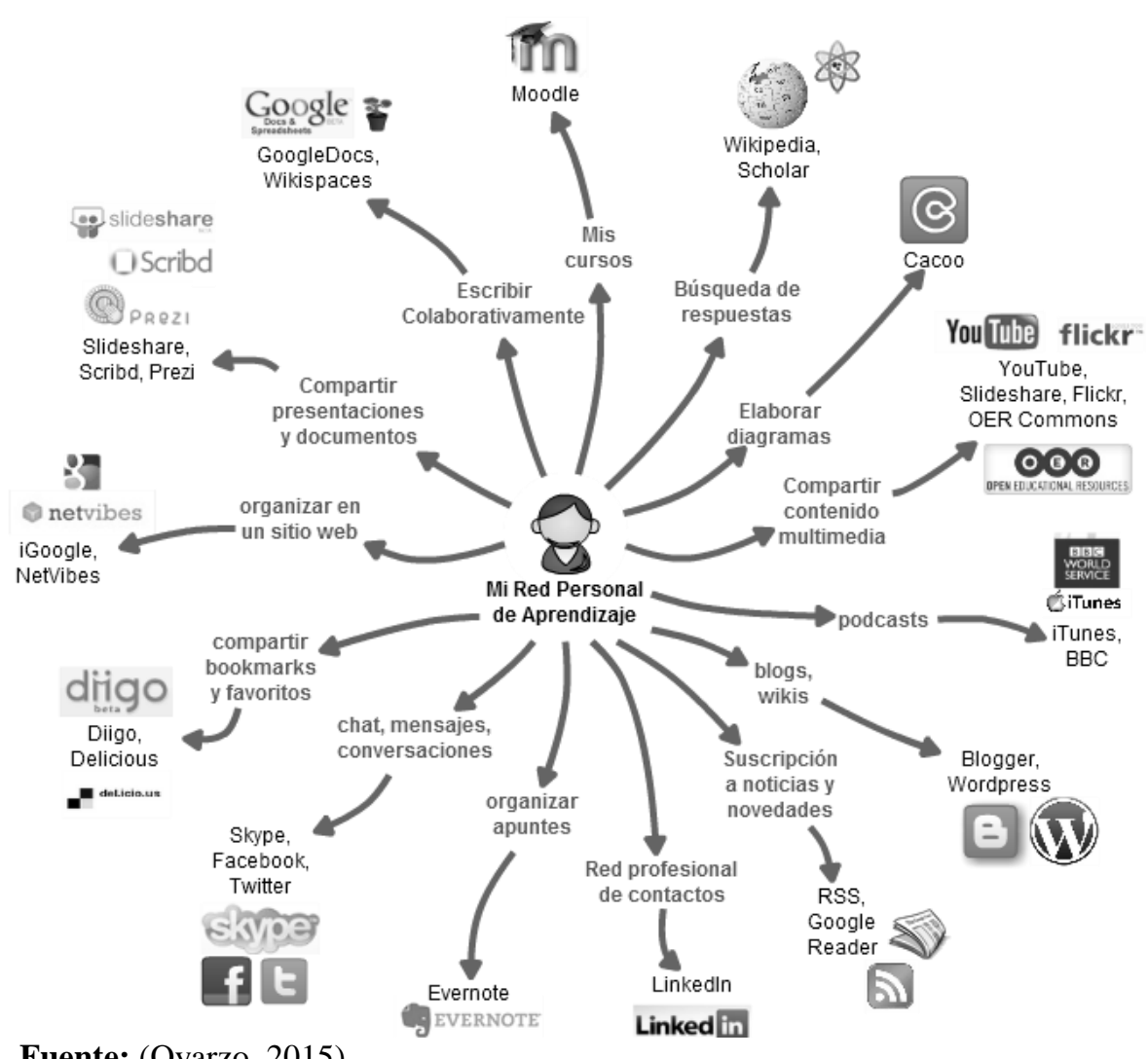

Fuente: (Oyarzo, 2015).

Figura 1. Recursos en línea de gestión autónoma y colaborativa.

\section{Objetivos}

Los objetivos formulados para la investigación:

- Conocer el nivel de aplicación de los recursos y herramientas en línea como bibliotecas virtuales, blogs, chat, correo electrónico, foros, plataformas virtuales, redes sociales, repositorios digitales, video conferencias en el aprendizaje autónomo universitario.

- Identificar los medios por los cuales se difunden los recursos y herramientas en línea que fomenten el aprendizaje autónomo universitario. 


\section{Metodología}

El estudio tiene como punto de partida el enfoque cuali-cuantitativo, tomando como referencia lo indicado por Pérez (1994) sobre la investigación educativa - como aquella modalidad de investigación destinada a evaluar los programas educativos en condiciones de rigor de cara a la mejora de las personas a las que se aplica. Las características principales de esta investigación son: practicidad y especificidad a realidades concretas, diversidad en los diseños de investigación ya que utiliza perspectivas desde el ámbito cuantitativo y cualitativo.

La modalidad de la investigación en una primera instancia es de carácter bibliográficadocumental; entendiéndose esta como el propósito de conocer, comparar, ampliar, profundizar y deducir diferentes enfoques, teorías, conceptualizaciones y criterios de diversos autores sobre una cuestión determinada, basándose en documentos (fuentes secundarias). Posteriormente, se aplicó la modalidad de campo, siendo este un estudio sistemático de los hechos en el lugar con la incorporación del trabajo autónomo en los estudiantes para tomar contacto en forma directa con la realidad, y obtener información de acuerdo con los objetivos del proyecto.

La técnica e instrumento aplicados, fueron la encuesta con un cuestionario estructurado, la investigación utilizó el análisis cualitativo y el análisis cuantitativo bajo el método de investigación transversal no experimental descriptiva. Conjuntamente la investigación se realizó en el lugar de los hechos "in situ". La estructuración del marco teórico, utilizó la investigación bibliográfica-documental, siendo insumo principal repositorios de información, que proveyeron argumentos y teorías útiles para el análisis de los datos y la construcción de las conclusiones. La investigación descriptiva, permitió tener resultados de los recursos y herramientas en línea en el aprendizaje autónomo universitario.

A continuación en la figura 2, se presenta el proceso metodológico utilizado.

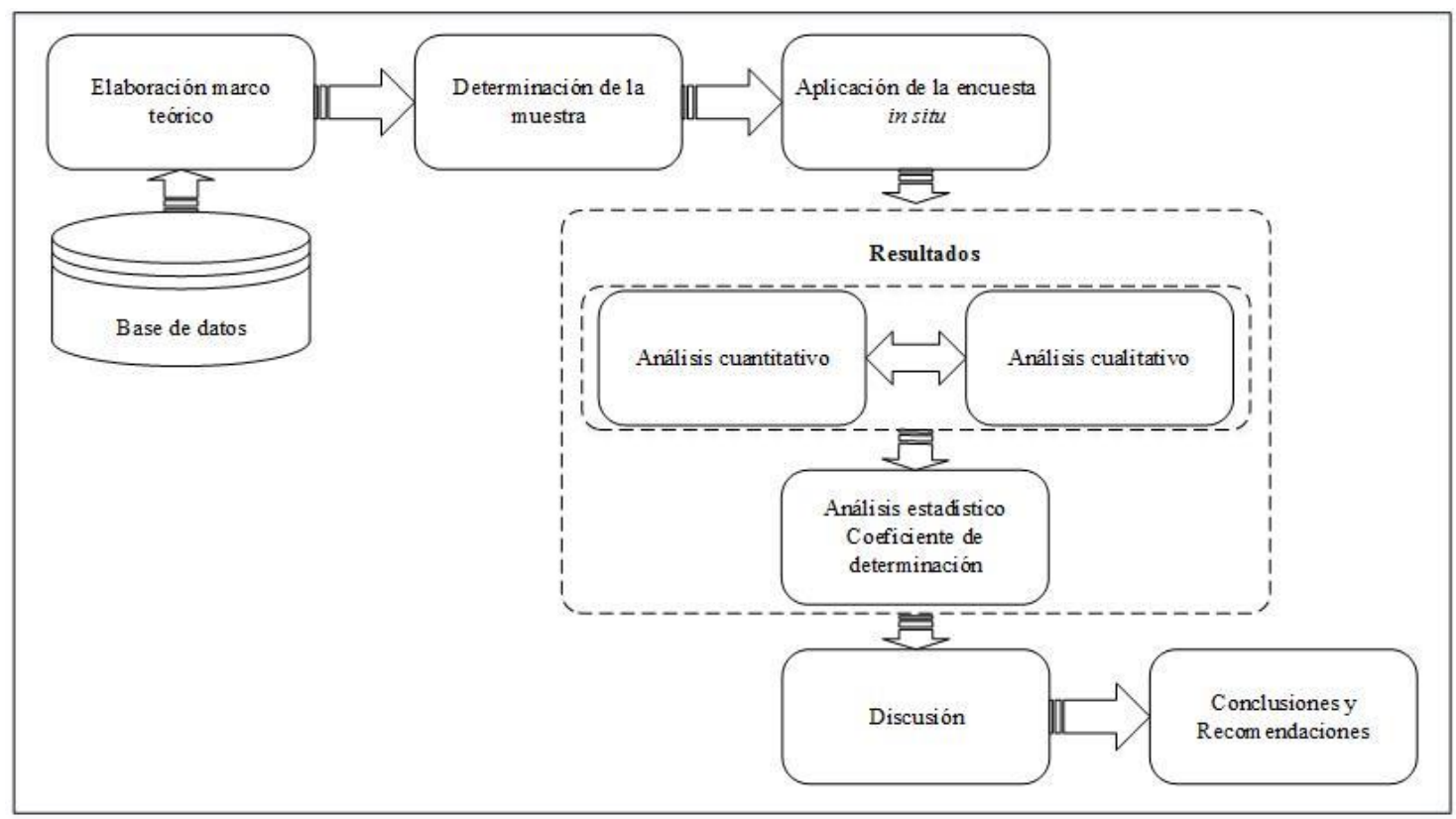

Figura 2. Proceso metodológico.

Los recursos tecnológicos en línea fortalecen el aprendizaje autónomo universitario. Luis Danilo Flores Rivera y Carlos Fernando Meléndez Tamayo. 
El instrumento utilizado fue la encuesta, que se aplicó a una muestra de una escuela de una universidad privada del Ecuador, utilizándose el muestreo probabilístico aleatorio simple.

Para el cálculo de las muestras se tuvo la siguiente fórmula, información y condiciones:

$$
\mathrm{n}=\frac{\mathrm{Z}^{2} \mathrm{PQN}}{\mathrm{Z}^{2} \mathrm{PQ}+\mathrm{Ne}^{2}}(\text { Ecu. 1) }
$$

$\mathrm{n}=$ Tamaño de la muestra.

$Z=$ Nivel de confiabilidad $90 \% \rightarrow 0,90 / 2=0,45 \rightarrow Z=1,645$

$\mathrm{P}=$ Probabilidad de ocurrencia $=0,5$

$\mathrm{Q}=$ Probabilidad de no ocurrencia 1- $0,5=0,5$

$\mathrm{N}=$ Población de estudiantes $=406$

$\mathrm{N}=$ Población de docentes $=41$

$\mathrm{e}=$ Error de muestreo 0, $1(10 \%)$

Luego de emplear la fórmula se obtuvieron las siguientes estimaciones, ver tabla 2:

Tabla 2

\begin{tabular}{ll} 
Muestra calculada & \\
\hline Muestra & N \\
\hline Estudiantes & 58 \\
Docentes & 26
\end{tabular}

Nota. Resultados del cálculo de la muestra con una $\mathrm{p}=0,5$.

La encuesta permitió conocer impresiones de los recursos y herramientas en línea concernientes a las actividades de aprendizaje autónomo. Para valorar el fortalecimiento de las herramientas en línea en el aprendizaje autónomo de los estudiantes, se comparó las calificaciones del primer y segundo parcial de un curso en particular de la universidad privada del Ecuador. El análisis utilizó el coeficiente de determinación "Una medida descriptiva del grado de la relación existente entre las variables" (Barrera Mellado, y otros, 2016)

Cabe indicar que la comparación del uso de herramientas en línea, se realizó con limitada práctica en el primer parcial y con mayor práctica en el segundo parcial.

\section{Resultados y discusión}

Los resultados de la tabla 3 , se pueden evidenciar que de la muestra de 58 estudiantes observados, los medios que consideran como valiosos para su aprendizaje autónomo son los siguientes: Plataformas Virtuales (PV) o Entornos Virtuales de Aprendizaje (EVA) $21 \%$, Bibliotecas Virtuales 20\%, Video conferencias 15\%, Blogs 11\%, Foros 10\%, Repositorios 10\%, Redes Sociales 8\%, Correo electrónico 4\%, Chat 2\%; de estos porcentajes se confirma que la preferencia de los estudiantes como herramientas que consolidan sus aprendizajes son las plataformas virtuales y bibliotecas virtuales; asi tambien, el análisis se relaciona con lo señalado por Moreno Martínez (2011), en el que menciona que los nativos digitales presentan otras formas de aprender y de acceder a la 
información para transformarla en conocimiento que les resultan muy motivadoras y funcionales a través de nuevos entornos virtuales y herramientas que ofrece la web 2.0 (...) redes sociales, blog, wikis, foros, webs de diversas temáticas, etc.

Tabla 3

Recursos y herramientas en línea que fortalecen al aprendizaje autónomo de estudiantes

\begin{tabular}{lcc}
\hline & $\begin{array}{c}\text { FRECUENCIA } \\
\text { ABSOLUTA }\end{array}$ & $\begin{array}{c}\text { PORCENTAJE } \\
\text { RELATIVO }\end{array}$ \\
\hline Bibliotecas virtuales & 33 & $20 \%$ \\
Blogs & 18 & $11 \%$ \\
Chat & 4 & $2 \%$ \\
Correo Electrónico & 7 & $4 \%$ \\
Foros & 17 & $10 \%$ \\
Plataformas o entornos virtuales & 35 & $21 \%$ \\
Redes Sociales & 13 & $8 \%$ \\
Repositorios digitales / base de datos universitarias & 16 & $10 \%$ \\
Video Conferencias & 25 & $15 \%$ \\
\hline Total & $\mathbf{1 6 8}$ & $\mathbf{1 0 0 \%}$ \\
\hline
\end{tabular}

Nota. Datos de la encuesta aplicada a 58 estudiantes.

Los resultados de la tabla 4, permite evidenciar que de la muestra de 26 docentes observados, sobre los medios que promueven el aprendizaje autónomo de los estudiantes en sus actividades académicas son los siguientes: Bibliotecas Virtuales 24\%, Plataformas Virtuales (PV) o Entornos Virtuales de Aprendizaje (EVA) 20\%, Repositorios Digitales 18\%, Correo electrónico 13\%, Foros 7\%, Video conferencias y Redes sociales $6 \%$, Blogs $4 \%$ y Chat $2 \%$; la preferencia de los docentes es el uso de Bibliotecas Virtuales, Plataformas Virtuales y Repositorios Digitales es similar al empleo de herramientas en línea que tienen los estudiantes; con la particularidad de que el docente en su trabajo académico consulta frecuentemente bases de datos universitarias. El análisis converge a lo señalado por Martínez León \& Ruiz Mercader (s.f.) donde destacan que entre las múltiples herramientas tecnológicas relacionadas con el aprendizaje en las organizaciones, así como entidades educativas, están las intranets, Internet, bases de datos, correo electrónico, chat, groupware, simuladores, redes de conocimiento, videoconferencia y espacios electrónicos.

Tabla 4

Recursos y herramientas en línea que utilizan los docentes

\begin{tabular}{lcc}
\hline & $\begin{array}{c}\text { FRECUENCIA } \\
\text { ABSOLUTA }\end{array}$ & $\begin{array}{c}\text { PORCENTAJE } \\
\text { RELATIVO }\end{array}$ \\
\hline Bibliotecas virtuales & 23 & $24 \%$ \\
Blogs & 4 & $4 \%$ \\
Chat & 2 & $2 \%$ \\
Correo Electrónico & 12 & $13 \%$ \\
Foros & 7 & $7 \%$ \\
Plataformas o entornos virtuales & 19 & $20 \%$ \\
Redes Sociales & 6 & $6 \%$ \\
Repositorios digitales / base de datos universitarias & 17 & $18 \%$ \\
Video Conferencias & 6 & $6 \%$ \\
\hline Total & $\mathbf{9 6}$ & $\mathbf{1 0 0 \%}$ \\
\hline
\end{tabular}

Nota. Datos de la encuesta aplicada a 26 docentes. 
En la figura 3, se muestra el resultado del uso de las herramientas en línea en el aprendizaje autónomo de los estudiantes universitarios. En los datos observados se analizó los rendimientos académicos del primer y segundo parcial. En el segundo parcial se experimentó un mayor uso de las herramientas en línea. Aplicando un software estadístico y calculando el coeficiente de determinación se obtuvo los siguientes resultados.

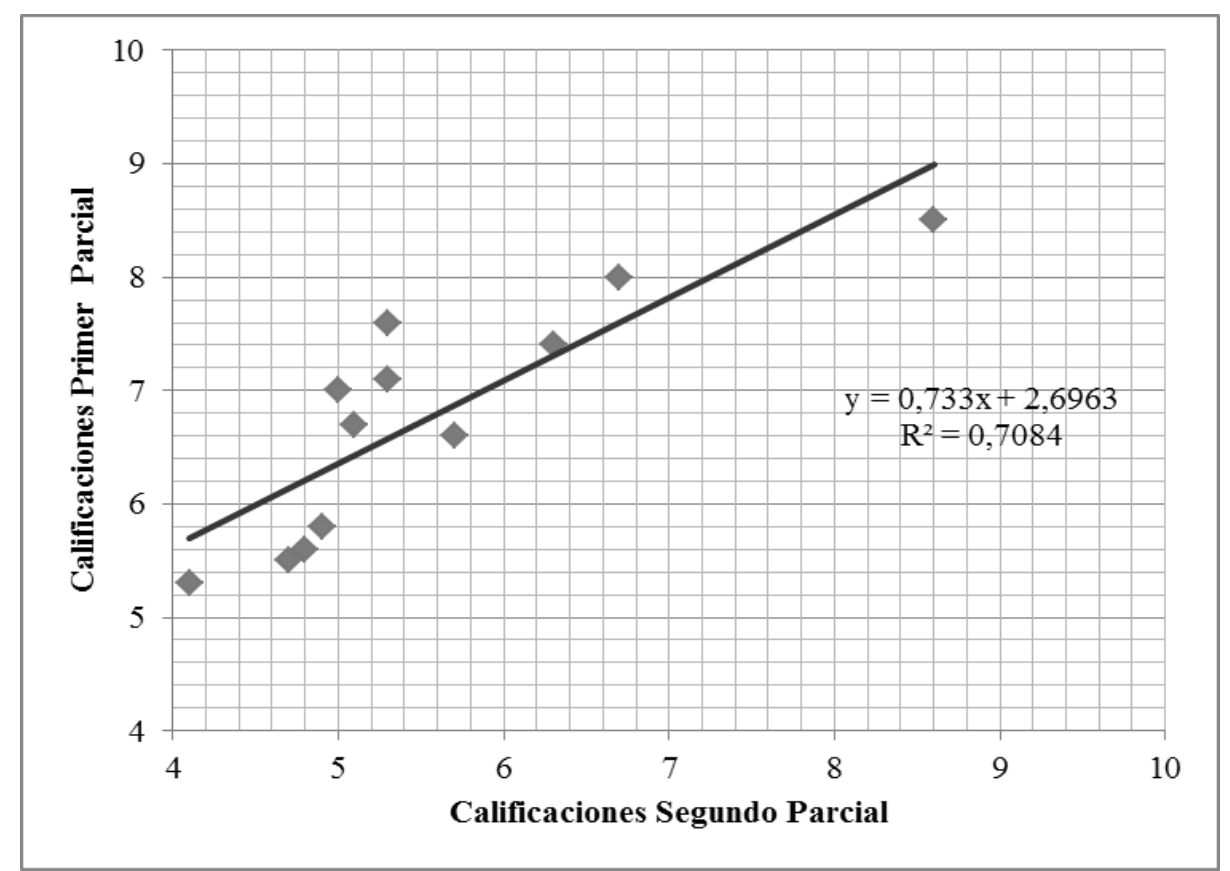

Figura 3. Análisis de determinación de rendimientos académicos.

Se estima una línea de tendencia creciente que infiere un incremento porcentual de las calificaciones, fortaleciéndose el rendimiento académico. El coeficiente de determinación obtenido es 0,7084 , por lo que las calificaciones del rendimiento académico tienen una variación del $71 \%$, que es significativo y determina una beneficiosa relación de las herramientas en línea con los aprendizajes obtenidos.

\section{Conclusiones y recomendaciones}

Una de las primeras conclusiones del trabajo es que las Tecnologias de la Información y Comunicación (TIC) pueden potenciar la articulación entre información, formación y reflexión siempre y cuando se utilicen como herramientas de comunicación e interacción entre personas, considerando que la incorporación de la información sea de forma crítica (Grande, Cañón, \& Cantón, 2016), (UNESCO, 2005) (Bautista, 2011).

La presente investigación especifica el nivel de aplicación de los recursos y herramientas en línea en función de las actividades académicas desarrolladas, las Biblioteca Virtuales, Plataformas Virtuales (PV) o Entornos Virtuales de Aprendizaje (EVA) y Repositorios Digitales son los de mayor aplicación, a diferencia de los Blogs y Chats los cuales son de menor aplicación. 
Por otra parte, los recursos tecnológicos en línea actualmente son parte esencial del aprendizaje universitario, puesto que fomentan la cultura investigativa, la innovación de aprendizajes y la retroalimentación (feedback) de conocimientos, es en ese sentido que los estudiantes y docentes apoyan la incorporación de los recursos y herramientas en línea a favor del aprendizaje universitario, puesto que han vivenciado las mejoras en el rendimiento académico (Flores Rivera, 2015), (Meléndez Tamayo, 2014), resulta necesario que el docente como actor del proceso educativo, debe orientar y motivar permanentemente el buen uso y/o aplicación de los recursos tecnológicos y herramientas en línea, para beneficiar el aprendizaje del estudiante.

El rendimiento académico se ve reflejado en la gestión del conocimiento por medio del aprendizaje adaptativo, en el que se consideran tres factores claves: el diagnóstico, la interacción y los resultados de las actividades. Se puede determinar en base de los resultados obtenidos de la investigación realizada: el aprendizaje adaptativo aplicado en las herramientas en línea y vinculado con el trabajo autónomo generan un incremento del $71 \%$ al rendimiento académico, fortaleciendo y potenciando el conocimiento entre los actores. (Demetra, 2017) (Instituto Tecnológico de Monterrey, 2014) (Cabero, 2001) (Lobato Fraile, 2006) (Martinez, 2004)

Presentación del artículo: 10 de junio de 2017 Fecha de aprobación: 15 de junio de 2017 Fecha de publicación: 30 de junio de 2017

Flores Rivera, L. D., y Meléndez Tamayo, C. F. (2017). Los recursos tecnológicos en línea fortalecen el aprendizaje autónomo universitario. RED, Revista de Educación a Distancia, 54. Consultado el (dd/mm/aaaa) en http://www.um.es/ead/red/54

\section{Financiación}

Esta investigación no ha recibido ninguna subvención específica de los organismos de financiación en los sectores públicos, comerciales o sin fines de lucro.

\section{Referencias}

Adell, J. (Noviembre de 1997). Tendencias en educación en la sociedad de las tecnologías de la información. EDUTEC: Revista electrónica de Tecnología Educativa(7).

Baelo Álvarez, R., \& Cantón Mayo, I. (10 de Noviembre de 2009). Las tecnologías de la información y la comunicación en la educación superior. Estudio descriptivo y de revisión. Iberoamericana de Educación, 1-12.

Los recursos tecnológicos en línea fortalecen el aprendizaje autónomo universitario. Luis Danilo Flores Rivera y Carlos Fernando Meléndez Tamayo. 
Barrera Mellado, I., Benito Maestre, M. S., Díaz Leno, M. S., Fernández Gómez, M. J., Galindo Villardón, M. P., Martín Rodríguez, J., y otros. (2016). Introducción a la Estadística. Salamanca: Universidad de Salamanca.

Bautista, A. (2011). Desarrollo tecnológico y educación. Educatio Siglo XXI, 435-438.

Cabero, J. (2001). Tecnología educativa. Barcelona: Paidós.

Cacheiro González, M. L. (2014). eBook Educación y Tecnología: Estrategias Didácticas para la Integración de las TIC. Madrid: UNED.

Cataldi, Z., Lage, F., \& Cabero, J. (Julio-Diciembre de 2010). La promoción de competencias en el trabajo grupal con base en tecnologías informáticas y sus implicancias didácticas. Pixel-Bit. Revista de Medios y Educación(37), 209-224.

CONESUP. (30 de Octubre de 2008). Reglamento de régimen academico del sistema nacional de educación superior. 26. Quito, Pichincha, Ecuador.

Corporación Intel. (9 de Mayo de 2015). Intel Educación y el Programa Intel Educar. Obtenido de Intel Educación y el Programa Intel Educar: http://www.intel.com/education/la/es/elementos/ccd/resources/herramientas_col aborativas_en_linea.pdf

D. K. (13 de marzo de 2017). Black Board Blog. Recuperado el 8 de junio de 2017, de Motivos para interesarse en el apredizaje adaptativo - Blog: http://blog.blackboard.com/motivos-para-interesarse-en-el-aprendizaje-en-lineaadaptativo/?lang=uki

De Pablos, J. (2007). Algunas reflexiones sobre las tecnologías digitales y su impacto a diferentes niveles sociales y educativos. En L. Aires, J. Azevedo, M. I. Gaspar, \& A. (. Teixeira, Comunidades Virtuais de Aprendizagem e Identidades no Ensino Superior (págs. 31-38). Lisboa: Universidade Aberta.

Ferroni, E., \& Velásquez, H. (Julio - Diciembre de 2005). Educación a distancia para salto académico. poliantea. Revista académica y cultural. Fundación Politécnico Grancolombiano Institución Universitaria,(4), 7-34.

Finch, A. (2010). A Task-based University EFL Program in Korea: Design, Implementation and Formative Evaluation. LAP LAMBERT Academic Publishing.

Flores Rivera, L. D. (13 de Agosto de 2015). El uso de herramientas en línea para fortalecer el aprendizaje autónomo de los estudiantes de la escuela de administración de empresas de la Pontificia Universidad Católica del Ecuador sede Ambato, período 2014 - 2015.[Tesis Maestría]. Universidad Nacional de Loja, Educación, El Arte Y La Comunicación. Loja: UNL.

$\overline{\text { Los recursos tecnológicos en línea fortalecen el aprendizaje autónomo universitario. Luis Danilo Flores }}$ Rivera y Carlos Fernando Meléndez Tamayo. 
García-Valcárcel, A., \& Tejedor, F. J. (1996). Perspectivas de las nuevas tecnologías en la educación. Madrid: NARCEA, S.A.

Giné Freixes, N. (2009). Cómo mejorar la docencia universitaria:El punto de vista del estudiantado. Revista Complutense de Educación, XX(9), 117-134.

Grande, M., Cañón, R., \& Cantón, I. (2016). Tecnologías de la información y la comunicación: evolución del concepto y características. International Journal of Educational Research and Innovation (IJERI), 218-230.

Gremmo, M.-J., \& Riley, P. (1995). Autonomy, self-direction and self access in language teaching and learning: The history of an idea. System, 23(2), 151-164.

Hill, T. E. (1991). Autonomy and Self-Respect. Cambridge: Cambridge University Press.

Holec, H. (1980). Autonomy and foreign language learning. Nancy: Centre de Recherches et d'Applications Pedagogiques en Langues. Council of Europe.

Instituto Tecnológico de Monterrey. (Julio de 2014). Recuperado el 6 de Junio de 2017, de http://www.sitios.itesm.mx/webtools/Zs2Ps/roie/julio14.pdf

Katsifli, D. (13 de Marzo de 2017). blog.blackboard.com. Recuperado el 8 de Junio de 2017, de blog.blackboard.com: http://blog.blackboard.com/motivos-parainteresarse-en-el-aprendizaje-en-linea-adaptativo/?lang=uki

Lindley, R. (1986). Autonomy. London: Macmillan.

Lobato Fraile, C. (2006). El estudio y trabajo autónomo del estudiante. En M. d. Díaz, Métodos y Modalidades de enseñanza centradas en el desarrollo de competencias. (págs. 191-223). Madrid: Alianza.

Majó, J., \& Marqués, P. (2002). La revolución educativa en la era internet. Barcelona: Cisspraxis.

Martínez León, I., \& Ruiz Mercader, J. (s.f.). upct.es. Obtenido de upct.es: http://www.upct.es/ economia/PUBLI-

INO/LAS\%20HERRAMIENTAS\%20DEL\%20APRENDIZAJE\%20EN\%20LA S\%20ORGANIZACIONES.pdf

Martínez, F. (1996). La enseñanza ante los nuevos canales de comunicación. En A. García-Valcárcel, \& F. J. Tejedor, Perspectivas de las nuevas tecnologías en la educación (págs. 101-136). Madrid: NARCEA S.A.

Martinez, J. (2004). Concepción del aprendizaje, metacognición y cambio conceptual en estudiantes universitarios. Barcelona: Universidad de Barcelona.

Meléndez Tamayo, C. F. (2014). La Web 2.0 como herramienta de apoyo docente. Ambato, Ecuador: Universidad Técnica de Ambato.

Los recursos tecnológicos en línea fortalecen el aprendizaje autónomo universitario. Luis Danilo Flores Rivera y Carlos Fernando Meléndez Tamayo. 
Moreno Martínez, N. M. (2011). Las TICS como herramientas para el desarrollo del aprendizaje autónomo del español como segunda lengua (12) en las a.t.a.l. Xii congreso internacional de tería de la educación (págs. 1-21). Barcelona: Universitat de Barcelona.

Moreno, R., \& Martínez, R. J. (2007). Aprendizaje autónomo. Desarrollo de una definición. Acta Comportamentalia, 15(1), 51-62.

Morrissey, J. (2008). El uso de TIC en la enseñanza y el aprendizaje. Cuestiones y desafíos. Las TIC: del aula a la agenda política (págs. 81-90). Buenos Aires: UNICEF ARGENTINA.

OCDE. (17 de Abril de 2002). oecd.org. Obtenido de oecd.org: http://www.oecd.org/internet/ieconomy/20627293.pdf

Oyarzo, J. (Mayo de 2015). PLE Diagrams. Obtenido de http://edtechpost.wikispaces.com/file/view/MyPLN.png/256240556/MyPLN.pn $\mathrm{g}$

Pemberton, R., Li, E. S., Or, W. W., \& Pierson, H. D. (1996). Taking Control: Autonomy in Language Learning. Hong Kong University Press.

Roblizo Colmenero , M. J., \& Cózar Gutiérrez, R. (Julio de 2015). Usos y competencias en tic en los futuros maestros de educación infantil y primaria: hacia una alfabetización tecnológica real para docentes. Píxel-Bit(47), 23-39.

Santiago Campión, R., \& Navaridas Nalda, F. (2012). La Web 2.0 en escena. Pixel-Bit. Revista de Medios y Educación, 19-30.

UNESCO. (2002). UNESCO Documents General Conference, Executive Board, 158162 EX and 31 C, End 1999-2001. París: UNESCO.

UNESCO. (2005). unesco.org. Obtenido de http://unesdoc.unesco.org/images/0014/001419/141908s.pdf 